\title{
150. The Effects of Chronic Destruction of the Inferior Olive upon Visual Modification of the Horizontal Vestibulo-Ocular Reflex of Rabbits
}

\author{
By Masao Ito and Yasushi MiYashita \\ Department of Physiology, Faculty of Medicine, \\ University of Tokyo, Bunkyo-ku, Tokyo \\ (Comm. by Yasuji Katsuki, M. J. A., Oct. 13, 1975)
}

Recent investigations of eye movements on human subjects,, 5$)$ monkeys, ${ }^{14), 18)}$ cats $^{17)}$ and rabbits ${ }^{3), 9), 10)}$ have revealed remarkable modification of the horizontal vestibulo-ocular reflex by visual stimuli. Two phases are discriminated of this effect; one is produced immediately and readily reversibly, while the other develops progressively and is maintained plastically. The former would represent an on-line device to correct erroneous performances of the vestibuloocular reflex by visual feedback, while the latter implies a sort of learning process with which the vestibulo-ocular system acquires the performance repeatedly improved by vision.6),7) Both of the immediate and progressive phases are impaired by extirpation of the cerebellar flocculus. ${ }^{9,10), 17), 18)}$ In accordance with these studies on eye movements, anatomical and electrophysiological investigations have demonstrated that the flocculus receives primary vestibular afferents $^{16)}$ as well as visual signals, ${ }^{11), 12)}$ and in turn sends out Purkinje cell impulses to certain second-order neurons of the vestibulo-ocular reflex arc. ${ }^{8)}$ Hence, it is postulated that the visual modification of the horizontal vestibulo-ocular reflex is effected through the cerebellar flocculus. ${ }^{6), 7)}$

In current theoretical considerations, ${ }^{1,13)}$ the inferior olive, the source of the climbing fiber afferent to the cerebellar cortex, is assumed to play an important role in the learning process which seemingly takes place in the cerebellar cortex. In fact, visual signals pass into the flocculus via the inferior olive. ${ }^{11)}$ It is interesting to see how the flocculus functions when the climbing fiber visual pathway is interrupted. In the present investigation, electrolytic lesions were placed at various antero-posterior levels of the inferior olive of rabbits, and the functional defects thereby produced were investigated by observing eye movements induced with combined vestibular and visual stimulation.

Adult albino rabbits of $3.0-4.0 \mathrm{~kg}$ were used. Under anesthesia by pentobarbitone sodium (Mintal, Tanabe, $40-50 \mathrm{mg} / \mathrm{kg}$ ), the dorsal 
atlanto-occipital membrane was opened and the lower medulla was exposed. A stainless needle, insulated except for the sharply pointed tip, was inserted into the medulla dorsoventrally, aiming at the right inferior olive. The antero-posterior level of inserting the needle varied from 0 to $4 \mathrm{~mm}$ rostral to the obex, and the laterality from 0.5 to $0.75 \mathrm{~mm}$ to the mid line of the medulla. Location of the needle at the depths of the inferior olive was guided by observing evoked potentials at the surface of the caudal pole of the cerebellar posterior lobe, while brief current pulses being applied to that needle as cathode, against anode served by a silver wire electrode placed on neck muscles. At those depths optimal for evoking climbing fiber responses at the cerebellar cortex, d.c. currents of $1 \mathrm{~mA}$ (tip positive) were passed through the needle for $60 \mathrm{sec}$. In order to avoid movements of the animal during passage of currents, the animal was paralysed by intravenous injection of Gallamin Triethiodide (Flaxidil), while the respiration was maintained through tracheal intubation. Out of 26 rabbits so prepared, 10 survived and recovered to the extent that they walked around on the floor of the laboratory. Experimental tests described below were accomplished within two months after the surgical operation. The 10 rabbits were then sacrificed and their brainstem was examined histologically. In 9 of these 10 rabbits, lesions were found to cover the inferior olive, rostrally in 5 and caudally in the other 4 .

To test the horizontal vestibulo-ocular reflex, the animal was rotated sinusoidally on the horizontal turntable either in darkness or under influences of visual stimuli.9) The visual stimuli were provided with a vertical slit light presented in front of the left eye. Movements of the left eye was observed by means of the closed circuit television system recently built in our laboratory (Batini, Ito, Kado, Miyashita and Yagi, to be published); a small piece of dried luminous paint $(0.2-0.3 \mathrm{~mm}$ in diameter) was implanted in the cornea just nasal to the pupil and under activation by low-level UV beam it served as the corneal mark. Displacement of this corneal mark around a fixed reference point on the monitor screen was converted into analog signals and registered by a pen recorder. Each period of the head rotation was divided into 18 bins and the eye position at each bin was averaged over 10 successive turns of head rotation. Averaged curves were subjected to a Fourier analysis by which the angular amplitude and phase angle of the eye rotation were calculated.

In 5 rabbits lesions covered nearly the whole area of the inferior olive on the transverse sections through its rostral portion, as seen in Fig. 1A and D. In these rabbits, the gain of the horizontal 

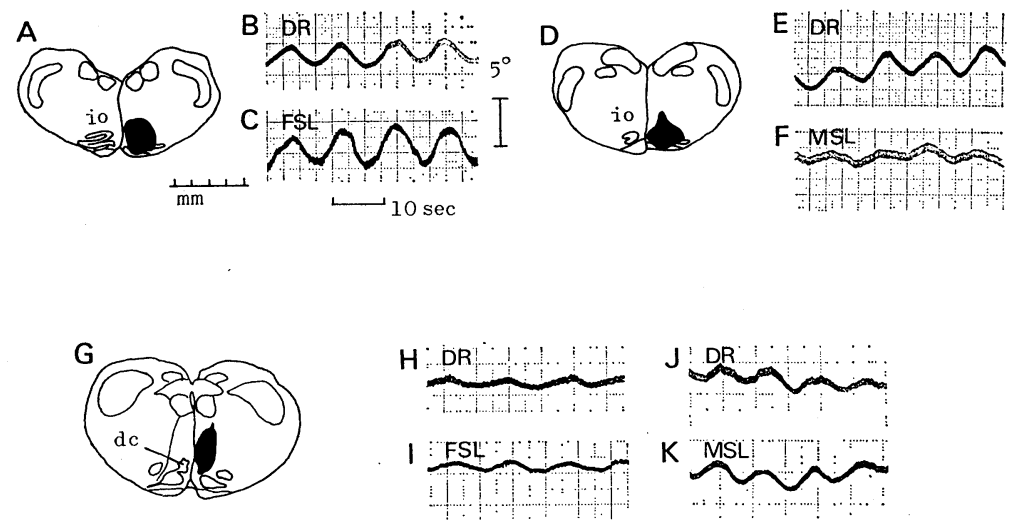

Fig. 1. Lesions in the inferior olive and the horizontal vestibulo-ocular reflex of rabbits $A$, transverse section of the medulla through the rostral portion of the inferior olive (io). On the the right side, the lesion is filled in black. $B$, horizontal rotation of the left eye measured in darkness (DR), while the rabbit was rotated sinuosidally on the horizontal plane at $0.1 \mathrm{~Hz}$ by $5^{\circ}$ peak-to-peak amplitude. C, taken immediately after B but with FSL presented. D-F, similar to A-D but in another preparation having the lesion at the rostral pole of the inferior olive and tested with MSL. G, third preparation with a caudally placed lesion. de, dorsal cap. H-I, similar to B-C, and $\mathrm{J}-\mathrm{K}$, to $\mathrm{E}-\mathrm{F}$, but for the preparation of $\mathrm{G}$.

vestibulo-ocular reflex, i.e., the amplitude ratio of eye vs. head measured in darkness, was $0.3-0.5$ over the amplitude range of 5-25 (peak to peak) for head rotation, as in normal rabbits. Frequency characteristics of the net vestibulo-ocular reflex were tested with $5^{\circ}$ head rotation at $0.03-0.5 \mathrm{~Hz}$ and also were similar to those in normal rabbits. On presenting the slit light fixed before the left eye (FSL), rotation of that eye was increased markedly in comparison with that measured in darkness (DR), as seen in Fig. 1B and C. This augmenting effect diminished as soon as the slit light was turned off. When the slit light was moved in phase with the head by an angular displacement twice as large as that of the head (MSL), it exerted a marked depressant action upon the eye rotation, as shown in Fig. 1E and $\mathrm{F}$. These observations indicated that, with lesions in the rostral portion of the inferior olive, dynamic characteristics of the horizontal vestibulo-ocular reflex and their dependence upon visual stimuli in terms of the immediate phase remained unchanged.

In the other 4 rabbits lesions placed relatively caudally involved the dorsal cap of the principal olive, as illustrated in Fig. 1G. In these rabbits, the gain of the horizontal vestibulo-ocular reflex was low, $0.1-0.3$, as measured with $5^{\circ}$ head rotation, and the effects of the slit light, either FSL or MSL, were scarce, as seen in Fig. 1H-K. 

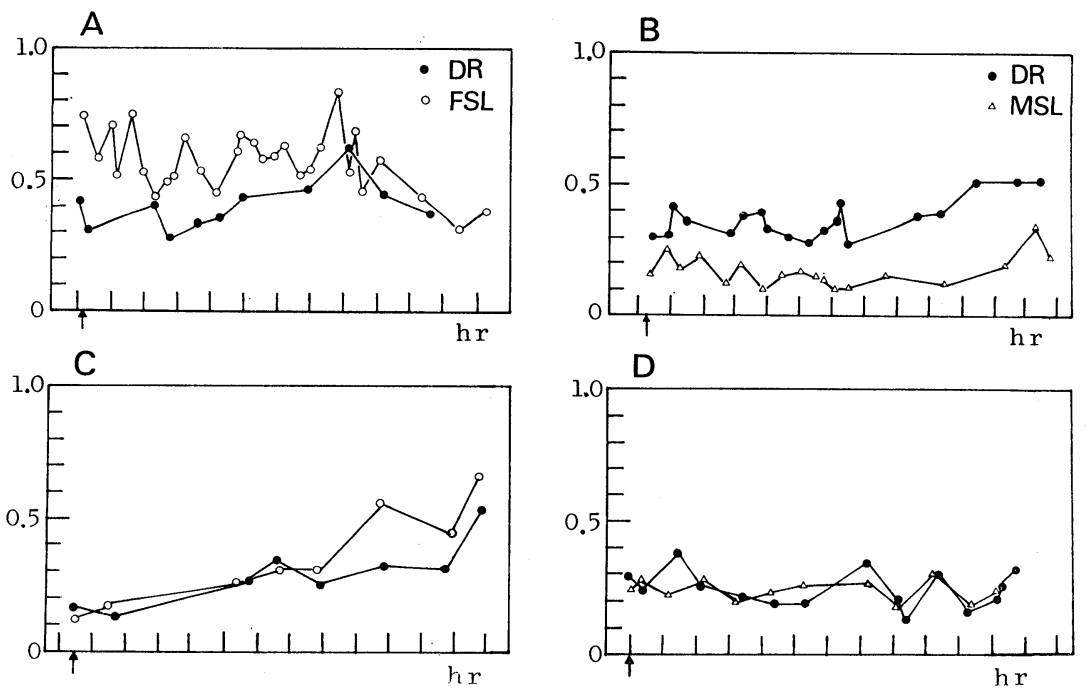

Fig. 2. Effects of the continuous application of the combined vestibular and visual stimulation Ordinates, gain of the horizontal vestibuloocular reflex measured with $5^{\circ}$ head rotation at $0.1 \mathrm{~Hz}$. A, for the preparation of Fig. 1 A-C. B, for that of Fig. 1 D-F. C and D, for that of Fig. $1 \mathrm{G}-\mathrm{K}$. Open circles in $\mathrm{A}$ and $\mathrm{C}$ plot the gain under influences of FSL and open triangles in B and D that under MSL. Closed circles in A-D indicate the gain measured in temporary darkness. Upward arrows on the abscissae mark the moment of onset of the continuous rotation.

These features resemble those obtained in the rabbits whose flocculus on the side of the tested eye had been destroyed chronically. ${ }^{9)}$

Three of the 5 rabbits with rostral lesions and all of the 4 with caudal lesions were further subjected to continuous rotation at $0.1 \mathrm{~Hz}$ for 12 hours, either with FSL or MSL presented. In normal rabbits, such a continuous rotation produced a progressive increase or decrease in the gain of the vestibulo-ocular reflex. ${ }^{10)}$ By contrast, in 16 (10 with FSL and 6 with MSL) of altogether 20 trials of the continuous rotation, no such progressive change occurred, as shown in Fig. 2A, B and D. In the remaining 4 trial with FSL, however, there was a progressive change but in a time course different from the normal ones; during the initial period of the continuous rotation, the gain increased very slowly and only slightly, but during the later period it increased rather steeply., as seen in Fig. 2C. This late effect was obtained in 2 of the 4 rabbits with caudal lesions, and contrasted to the initially rapid and later slower gain increase in normal rabbits. ${ }^{10)}$

The 7 rabbits tested with continuous rotation were then anesthetized by intravenous injection of pentobarbitone sodium. The 
optic chiasm was stimulated electrically, while a glass microelectrode was inserted into the left flocculus posteriorly through the paraflocculus. ${ }^{4)}$ The stimulation was adjusted to evoke responses in the visual cortex of the cerebrum, yet no visible field potentials were produced in the left flocculus. This indicated that the visual pathway through the inferior olive had been interrupted by the lesions, either rostral or caudal.

Since interruption of the climbing fiber visual pathway with rostral lesions did not impair the immediate modification of the horizontal vestibulo-ocular reflex by visual stimuli, this immediate visual effect should be mediated by another pathway. The likely candidate is the mossy fiber pathway which conveys visual signals to the flocculus. ${ }^{12)}$ The fact that the caudal lesions of the inferior olive involving the dorsal cap resulted in decrease of the gain and loss of the immediate visual effect may be related to the recent finding that the olivocerebellar neurons mediating visual signals to the flocculus are located caudally in the dorsal cap. ${ }^{2), 15)}$ Damage of these cells might result not only in deprivation of visual signals to the flocculus mediated by them, but also in disability of the cortical neuronal networks. With either rostral or caudal lesions in the inferior olive, the progressive phase of the visual modification of the horizontal vestibulo-ocular reflex was lost, or it took an unusual time course. This observation supports the hypothesis that the inferior olive plays an important role in the learning process of the cerebellar cortex. ${ }^{1), 13)}$

\section{References}

1) Albus, J. S.: Math. Biosci., 10, 25 (1971).

2) Alley, K., Baker, R., and Simpson, J. I.: in press Brain Res.

3) Baarsma, E. A., and Collewijn, H.: J. Physiol., 238, 603 (1974).

4) Ghelarducci, B., Ito, M., and Yagi, N.: Brain Res., 87, 66 (1975).

5) Gonshor, A., and Melvill-Jones, G.: J. Physiol., 234, 102 (1973).

6) Ito, M.: Brain Res., 37, 387 (1972).

7) - : In Neurosciences, III, MIT Press, 293 (1974).

8) Ito, M., Nisimaru, N., and Yamamoto, M.: Brain Res., 60, 238 (1973).

9) Ito, M., Shiida, T., Yagi, N., and Yamamoto M.: Brain Res., 65, 170 (1974).

10) Ito, M., Shiida, T., Yagi, N., and Yamamoto, M.: Proc. Japan Acad., 50, 85 (1974).

11) Maekawa, K., and Simpson, J. I.: J. Neurophysiol., 36, 649 (1973).

12) Maekawa, K., and Takeda, T.: Brain Res., 98, 590 (1975).

13) Marr, D.: J. Physiol., 202, 437 (1969).

14) Miles, F. A., and Fuller, J. H.: Brain Res., 80, 512 (1974).

15) Mizuno, N., Nakamura, Y., and Iwahori, N.: Brain Res., 77, 385 (1974).

16) Precht, W., and Llinás, R.: Exp. Brain Res., 9, 30 (1969).

17) Robinson, D. A.: In Basic Mechanisms of Ocular Motility and Their Clinical Implications. Pergamon, Oxford (in press).

18) Takemori, S., and Cohen, B.: Brain Res., 72, 203 and 213 (1974). 